solution qui ne coagulait pas le lait non acidulé même au bout de $3 \frac{1}{2}$ heures à la temṕrature la plus favorable pour la coagulation.

Q xoique l'auteur au bout de son article ait déclaré : " Es war aber keineswegs meine Absicht eine erschöpfende Uebersicht über diese interessante Frage zu geben » (1), une expérien si importante exigeait plus de développəment; aussi l'absence complète des indications teshniques nézessaires en dépréc e la valeur. C'est ainsi que la quantité de $p э p$ sine utilisée n'est pas indiquée; on ne dit pas de quelle manière a été obtenue le caillé. Si c'est par acidulation forte du lait, alors cela change tout à fait les conditions physico-chimiques de la maturation du fromage. Il reste à supposer que l'auteur se servait d'une culture très active de microorganismes lactiques, qui se sont montrés capables de pəptoniser à un degré suffisant les albumines du fromage malgré la quantité évidemment insuffisante de lab.

Le rôle des microorganismes lactiques dans le processus de la maturation du fromage se présentait à diverses reprises comme thème des expériences. Mais ce n'est que récemment qu'on a réussi à montrer que les bactéries lactiques, qui appartiennent au groupe Streptococcus lactis, possèdent une capacité assez considérable de désagréger la caséine. Ce mérite appartient au bactériologiste suédois Chr. BARTHEL [22] qui a publié en 1915 un article sous le titre : "Das kaseinspaltende Vermözen von zur Gruppe Streptococcus lactis gehörenden Milchsäurebakterien » (2).

(A suivre.)

\title{
QUELQUES NOTES DE LAITERIE SUR L'ALGÉRIE
}

\author{
par C. BAN DANOU.
}

Docteur Vétérinaire, Ancien Répátıteur de Zootıchnie à l'Ecole Nationale d'Agric Itture de Montpellier; Service de l'Elevage dy l'Algérie à Miliana.

Dans les steppes du sud algérien où $n$ madisent les 9/10 du cheptel ovin, le lait de brebis sert en partie à l'élevage des jeunes, mais surtout à l'alimentation des tribus pastorales. Celles-ci en extraient d'abord le beurre (,) et consomment le petit-lait après fermentation lactique, soit en nature, soit sous forme de caséine desséchée ou klila.

Lors des années pluvieuses, lorsque l'herbe ne manque pas dans les steppes, les agnelages s'échelonnent sur les quatre saisons :

$\begin{array}{lll}R ' b i i & \text { agnelages de printemps } \\ \text { Saï i } & \text { " } & \text { d'été } \\ \text { Kherfi } & \text { " } & \text { d'automne } \\ \text { Chetoui } & \text { " } & \text { d'hiver }\end{array}$

(1) Mais je n'ai, en aucune manière, l'intention de donner un aperçu définitif sur cette question intéressante.

(2) La capacité de désagréger la caséine des bactéries acido-lactiques du groupe Strep. tococcus lactis.

(3) "Industrie beurrière ehez les nomades des Hauts-Plateaux ", par BeN Danou (Bulletin de l'Office Colonial de l'Algérie, année 1911). 
Nous avons souligné à dessein, les agnelages de printemps et d'automne, qui sont les plus importants.

Le lait de brebis ne fait donc pas défaut; mais en prévision des périodes sèches, l'excès de petit-lait sert à préparer la klila ou caséine desséchée, de réserve.

Voici comment cette "klila » est préparée :

Le lait de brebis, tiré dans des réeipients en alfa est laissé à luimême pendant 24 ou 48 heures selon le degré de température. Sa richesse en caséine fait que la montée de la crème est assez lente et souvent la.fermentation lactique a eu le temps de s'installer et de produire un caillé assez ferme, avant que toute la matière grasse du lait soit montée à la surface de sorte que les caillots emprisonnent encore dans leurs mailles une assez notable quantité de matière grasse. C'est pourquoi le petit-lait des pasteurs nomades est toujours plus riche en beurre que le petit-lait sorti des écrémeuses.

Quoi qu'il en soit, dès que la crème a été prélevée pour la confection du beurre ou zebda, ce qui reste du lait représente un sous-produit encore riche en matière grasse et renfermant la totalité de tous les autres éléments du lait, c'est-à-dire des protéines, des matières grasses retenues dans les caillots, des phosphates et autres sels, du lactose, de l'acide lactique. C'est tout cela que le nomade ne laisse pas perdre et qu'il saura utiliser en l'absorbant sous forme liquide (leben) et en en extrayant une caséine de conserve qui servira à remplacer la viande dans les préparations culinaires, sous la tente des populations des oasis ou des steppes.

Cette caséine est ainsi obtenue : le lait caillé, débarrassé de sa crème, est ehauffé sur un foyer; les caillots se forment plus nombreux et plus consistants. On passe le tout sur un linge fin et l'amas cailleboteux est exposé au soleil.

Après dessic ation jusqu'à consistance pierreuse, les blocs sont finement broyés et pétris avec le sérum avec:

$$
\begin{aligned}
& \text { des dattes } \\
& \text { dn beurre } \\
& \text { des épices } \\
& \text { de la farine d'orge }
\end{aligned}
$$

la pâte est ensuite divisée en petites boulettes que l'on expose encore au soleil et c'est la klila ou biscuit de guerre, caséine de réserve, très appréciée des nomades.

La klila sous forme exclusive de blocs pierreux, sans addition de dattes, est l'objet de transactions entre les populations du sud et les populations des oasis, ar dans les oasis, si quelques privilégiés entretiennent des unités ou quelques faibles lots de brebis doumanes (1), la Toulouse). 
population manque totalement de viande. Et la caséine desséchée tient lieu de "protéine viande " dans certaines préparations culinaires.

Les populations nomades' qui parcourent le sud, ainsi que les sédentaires fixés dans les oasis, ont appris, au choe des réalités, à être économes en matière alimentaire. Elles savent tirer le maximum de ce que la nature leur dispense avec tant de parcimonie, en ces régions deshéritées. Et il est heureux que la siccité considérable de l'atmosphère, la vive lumière, et les oxydations permettent la conservation prolongée de denrées les plus périssables. Sans eela on ne comprendrait pas comment, dans les conditions d'hygiène les plus surprenantes, les nomades ne voient jamais leur lait se détériorer. Et pourtant l'eau, si largement utilisée en Europe, est une denrée rare, précieuse aussi, dans le Sud, et surtout réservée pour l'usage interne (1).

Nous noterons enfin que nos brebis sont admirablement adaptées et par les qualités de leurs toisons et par un entraînement plusieurs fois séculaire et devenu héréditaire pour lutter contre la deshydratation.

\section{Le Lait en thérapeutique chez les Indigènes}

Du Nord à l'extrême sud, nos indigènes, depuis un temps immémorial, utilisent le lait humain dans le traitement des affections des yeux surtout chez les enfants.

Les femmes qui allaitent, complètent la toilette de l'enfant en lui projetant leur lait directement de la mamelle dans les yeux maintenus ouverts.

Nombre de mères présentent systématiquement leurs enfants à des nourrices réputées comme ayant un lait actif. $€ t$ celles-ci se prêtent de bonne grâce et cela sans aucune rétribution à ce qu'on attend d'elles. Le mamelon est dirigé vers l'œil dont les paupières sont maintenues écartées et le jet lacté projeté à petite distance sur le globe oculaire.

Et comme, chez les primitifs, la superstition ne perd jamais ses droits, il est de croyance courante que le lait le plus actif provient des nourrices dont l'enfant est une fille. Lorsque le nourrisson est un garçon, le lait de la mère serait moins actif. Nous notons en passant ces observations.

Pour le traitement des plaies qui suppurent, le lait est également considéré par les ind gènes, comme un remède puissant. Ils l'utilisent en application directe au moyen de mie de pain trempée dans le lait ou de compresses.

On voit qu'il n'y a rien de nouveau sous le soleil. La science, en beaucoup c'e cas, ne fait qu'expliquer le pourquoi.

(1) Les récipients sont en alfa, les peaux de bouc servent de barattes. (Note de l'auteur). 